\title{
Reconstrucción del complejo areola-pezón: revisión de 60 casos
}

\section{Nipple-areola complex reconstruction: revision of 60 cases}

Fernández García, R.*, Fernández Delgado, J .**, Martínez Méndez, J .R.*, Bravo Brañas, E.**, García Redondo, M.*, Sordo Miralles, G.*, Casado Pérez, C.***

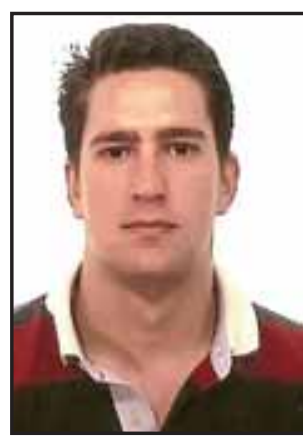

Fernández García, R.

\section{Resumen}

La creación del complejo areola-pezón, CAP, constituye el último tiempo de la reconstrucción mamaria, transformando la reconstrucción del montículo mamario en una mama. Debemos considerar pues la reconstrucción de la areola y el pezón, como la culminación de la reconstrucción de la mama.

Existen numerosas técnicas descritas para la reconstrucción del CAP. El objetivo de este estudio es determinar el grado de satisfacción psicológica de las pacientes tras la misma.

El presente estudio fue diseñado como una revisión clínica retrospectiva de 60 pacientes. Tras la revisión de las historias clínicas, las pacientes fueron entrevistadas procediéndose a la realización del cuestionario.

El aspecto a cambiar más deseado fue la falta/pérdida de proyección del pezón. No obstante, el $22 \%$ de las pacientes respondieron que no cambiarían nada acerca de su reconstrucción.

La satisfacción acerca de la reconstrucción del montículo mamario fue excelente o buena para el $68 \%$, normal para el $23 \%$ y pobre para el $9 \%$. En cambio, para la reconstrucción del complejo areola-pezón fue excelente o buena para el $50 \%$, normal para el $45 \%$ y pobre para el $5 \%$.

No se encontraron diferencias significativas entre las diferentes técnicas en función del tiempo transcurrido entre el momento de la mastectomía y el tercer tiempo de la reconstrucción $(\mathrm{p}=0,06)$.

La técnica de la donación contralateral de pezón fue la que ofreció una mayor satisfacción (2,67 puntos) y proyección (7,23 puntos). A pesar de las diferencias en sus medias, no se demostró ninguna diferencia estadísticamente significativa.

Teniéndo en cuenta la técnica usada para la reconstrucción de la areola, la técnica de la donación-injerto de piel inguinal fue la que ofreció mayor satisfacción (3 puntos) y coloración (8,57 puntos). El estudio estadístico objetivó diferencias estadísticamente significativas $(\mathrm{p}=0,01)$

Entre las distintas técnicas de reconstrucción del pezón no hay ninguna cuyos resultados se sobrepongan a las demás, pero sí una vez que se reconstruye la areola siendo la técnica que más satisface la del injerto inguinal. A pesar de ello, el aspecto más notable a mejorar está en la reconstrucción del pezón, dada la frecuente disconformidad de las pacientes con la proyección conseguida a lo largo del tiempo por la posible reabsorción del mismo.

Palabras clave Reconstrucción mamaria. Complejo areola pezón

Código numérico 5214-5212
The creation of the nipple-areola complex is the latest time in breast reconstruction, transforming the reconstruction of the breast mound into a real breast. We have to consider the reconstruction of the areola and the nipple as the culmination of breast reconstruction.

There are a lot of documented techniques for nipple-areola complex reconstruction. The aim of this study is to determine the grade of psychological satisfaction of patients after this reconstruction

This study was designed as a retrospective clinic review of 60 patients. After reviewing medical histories, the patients were interviewed and asked to complete a questionnaire.

The most common desired aspect was to correct the absence/lost of nipple projection. However, $22 \%$ of patients answered they would not change anything regarding their reconstruction.

The satisfaction with the mammary mound was excellent or good for $68 \%$, normal for $23 \%$ and poor for $9 \%$. On the other hand, satisfaction for the nippleareola complex reconstruction was excellent or good for $50 \%$, normal for $45 \%$ and poor for $5 \%$.

There were no statistical differences among the different techniques depending on the time between the mastectomy intervention and the third reconstruction $(\mathrm{p}=0,06)$.

For nipple reconstruction, the contralateral nipple donation technique offered more satisfaction ( 2.67 points) and projection ( 7.23 points). In spite of the differences in their means, there were no statistically significant differences.

Taking into account the technique used for the areola reconstruction, the donation-graft of inguinal skin was the one that offered more satisfaction ( 3 points) and better coloration ( 8.57 points). With the statistical study there were obtained significant statistical differences $(\mathrm{p}=0,01)$.

Among the different techniques for nipple reconstruction there wasn't anyone whose results overlapped the others, but when the areola was reconstructed there were some, being the inguinal grafting the technique which satisfies more. In spite of this, the aspect which most needed to improve is nipple reconstruction because many patients were unhuppy with the obtained projection and its reabsorption over time.

Key words Breast reconstruction. Nipple-areolar complex

Numeral Code $\quad 5214-5212$

\footnotetext{
* Médico Interno Residente.

* Cirujano Plástico.

* J J efe de Servicio
}

Servicio de Cirugía Plástica, Estética y Reparadora. Hospital Universitario La Paz. Madrid. España. 


\section{Introducción}

La creación del complejo areola-pezón, CAP, constituye el último tiempo de la reconstrucción mamaria, transformando la reconstrucción del montículo mamario en una auténtica mama. La presencia del complejo areola-pezón posibilita a la paciente para hablar de una mama reconstruida completa y no solo de un volumen mamario (1), dado que este último paso aporta una apariencia natural, especialmente cuando la paciente está desnuda. Por todo ello, debemos considerar la reconstrucción de la areola y el pezón como la culminación de la reconstrucción de la mama (2-7).

Para asegurar un montículo mamario subyacente estable, la reconstrucción del CAP debe retrasarse por lo menos 2 meses hasta que la mama haya asumido su forma y posición finales. Por ello, la reconstrucción del CAP se realiza en algunos casos en el segundo tiempo quirúrgico, cuando se ha hecho una reconstrucción mamaria inmediata, (RMI), pero en la mayor parte de los casos se realiza en un tercer tiempo quirúrgico (2-10).

Existen numerosas técnicas descritas para la reconstrucción del CAP (8-12). Por ello, el objetivo de este estudio es determinar el grado de satisfacción psicológica de las pacientes independientemente del tipo de reconstrucción mamaria practicada, así como en función de la técnica realizada. Asímismo, se evaluaron los determinantes clínicos que influyen en el resultado final.

\section{Pacientes y método}

El presente estudio fue diseñado como una revisión clínica retrospectiva de 60 pacientes a las que se les reconstruyó tanto el montículo mamario como el complejo areola-pezón en el Servicio de Cirugía Plás-

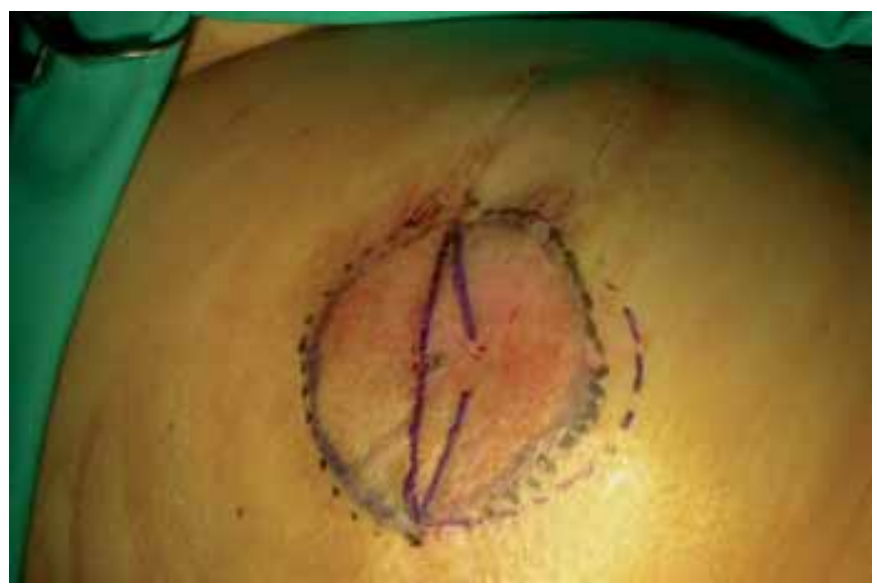

Fig. 1. Diseño intraoperatorio del colgajo en cola de delfín. tica, Estética y Reparadora del Hospital Universitario La Paz (HULP) de Madrid, España, desde Enero 2003 hasta Diciembre 2005. En el mismo tan solo se incluyeron las pacientes que requirieron reconstrucción mamaria por causa neoplásica.

Una vez realizada la revisión de las historias clínicas para la valoración de la técnica quirúrgica empleada, las pacientes fueron entrevistadas en persona y se procedió a la realización del cuestionario. Todas las pacientes vivas a las que se reconstruyó la mama por cirujanos adjuntos del Servicio de Cirugía Plástica del HULP fueron invitadas a participar en el estudio. Tres pacientes no pudieron ser localizadas, y de las restantes 57 pacientes, todas decidieron participar.

Tras completarse los cuestionarios, se obtuvieron fotografías clínicas usándose para ello distancias standard con cámara digital de 4 millones de pixels.

Los cuestionarios evaluaron la satisfacción con la reconstrucción del montículo mamario; la satisfacción con la reconstrucción del complejo areola-pezón; cuál era el aspecto con el que menos conforme se encontraban sobre la reconstrucción del CAP; si la paciente volvería a reconstruirse la mama en caso de ser necesario y si la reconstrucción del CAP era absolutamente necesaria. Los distintos parámetros fueron entonces calificados estadísticamente con el objetivo de clarificar el grado de satisfacción.

El grado de satisfacción individual fue calificado evaluándose la satisfacción del montículo mamario independientemente de la del CAP. El sistema se graduó siguiendo el siguiente criterio: $3=$ Excelente/ buena, $2=$ Normal, y 1=Pobre. A su vez, a la hora de elegir entre los aspectos con los que se encontraban con mayor disconformidad, se les aportó la posibilidad de elegir varios de los aportados.

El estudio estadístico fue llevado a cabo por el análisis de la varianza y el test de Student.

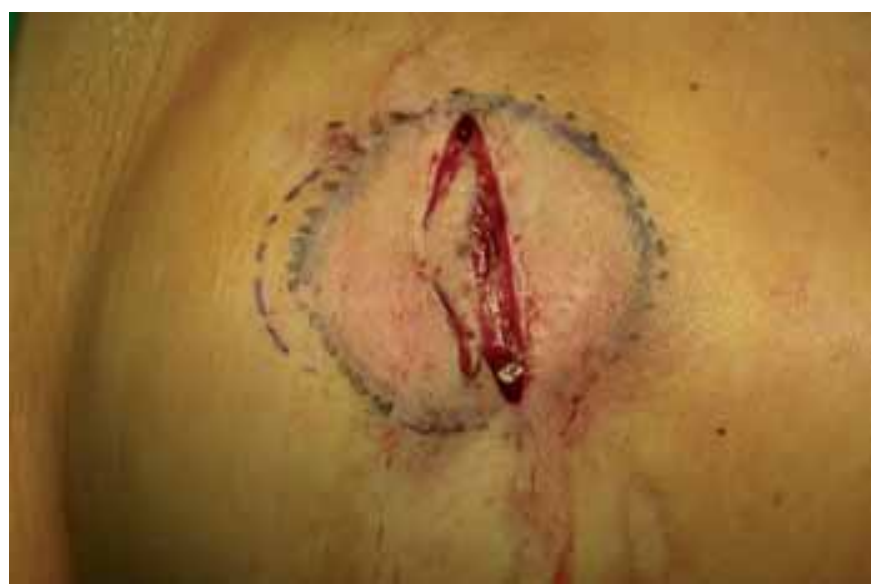

Fig. 2. Pasos intraoperatorios secuenciados de la técnica del colgajo en cola de delfín: Desepitelización del colgajo tipo delfín. 


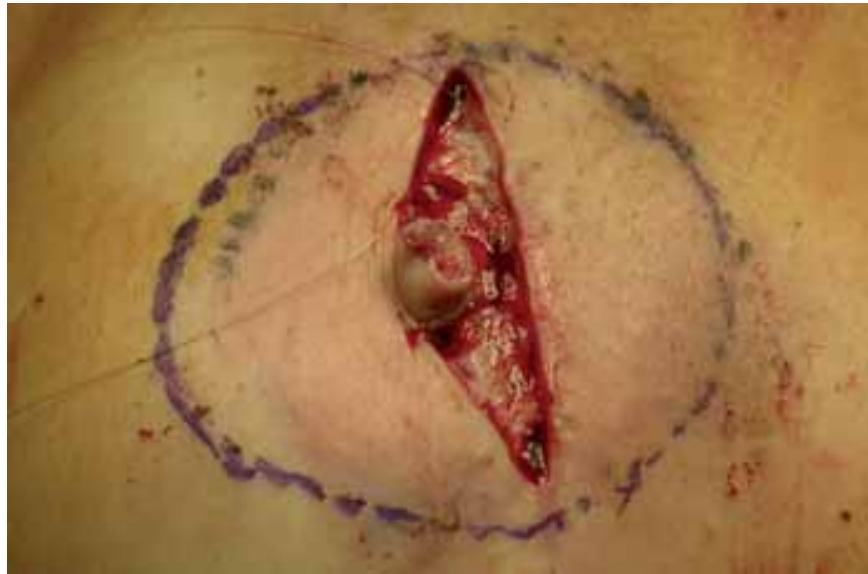

Fig. 3. Plegamiento del colgajo sobre sí mismo conformándose el futuro pezón.

\section{Resultados}

Mediante la revisión de historias clínicas se evidenció el tipo de reconstrucción mamaria llevada a cabo. En el presente estudio tan solo se incluyeron las pacientes a las cuales se les practicó reconstrucción de areola y pezón. A 30 pacientes se les implantó un expansor seguido de recambio por prótesis (49\%), a 23 pacientes se les practicó reconstrucción de mama inmediata (38\%), de las cuales 22 fueron con prótesis y 1 caso con expansor y prótesis; a 4 pacientes se les reconstruyó mediante colgajo de músculo dorsal ancho junto con prótesis $(6 \%) ; 1$ paciente tan solo requirió de colgajo de dorsal ancho aislado (1,5\%); 1 paciente se reconstruyó con prótesis aislada $(1,5 \%)$ y 2 pacientes se reconstruyeron por métodos alternativos (3\%) (en estos casos la técnica de reconstrucción no se pudo recoger por no estar claramente descrita en la historia clínica, bien por tratarse de técnicas personales o por omisión de datos).

Respecto al tipo de reconstrucción del pezón, 42 pacientes fueron reconstruidas mediante colgajos locales (70\%); 21 de éstos siguieron la técnica en "cola de delfín” (35\%) (Fig. 1-6), 13 el colgajo C-V (22\%) y 8

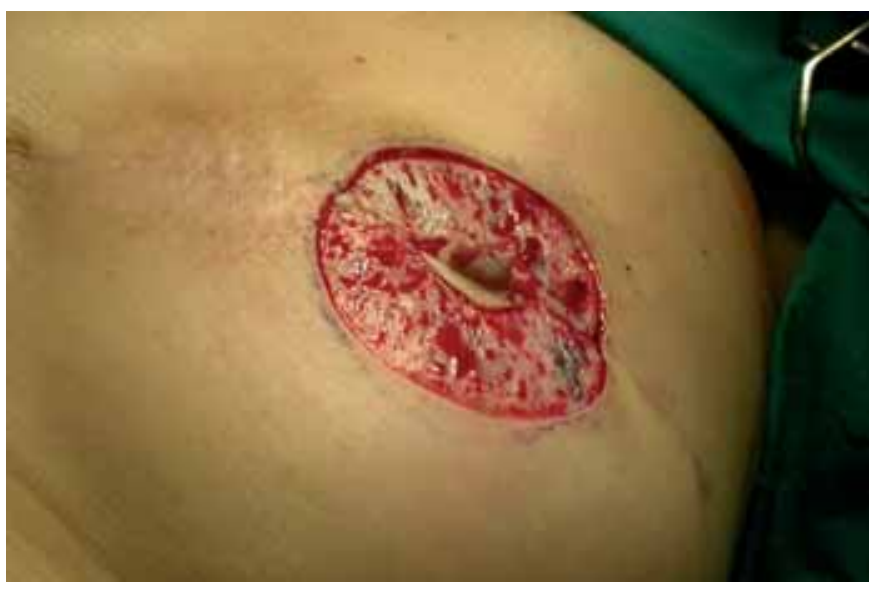

Fig. 5. Lecho areolar una vez desepitelizada la piel circundante.

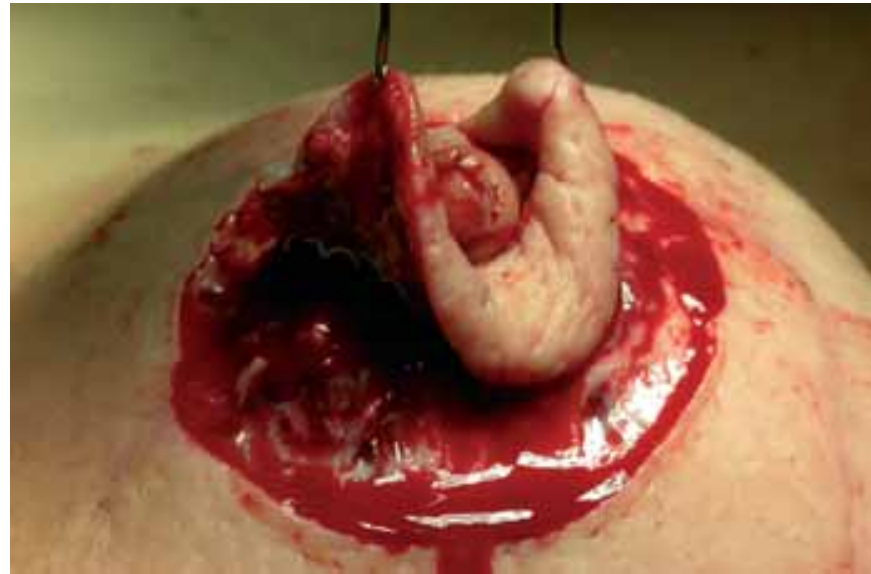

Fig. 4. Desepitelización areola.

otra técnica alternativa no identificada (13\%). Quince pacientes fueron reconstruidas mediante donación del pezón contralateral $(25 \%)$. Analizándose el número de intervenciones necesarias para la reconstrucción del pezón, se objetivó que tan solo 2 pacientes requirieron de dos procedimientos. En el resto, con tan solo una intervención quirúrgica fue suficiente $(97 \%)$.

El proceso de reconstrucción de la areola se practicó en 33 pacientes mediante autoinjerto local de la piel circundante al neopezón (55\%) (Fig. 2 - 7). En 9 casos fue llevado a cabo mediante donación de areola contralateral (15\%). En 10 casos se procedió a la toma de piel autóloga (16\%), 8 de las veces inguinal y 2 de ellas axilar. En 4 de los casos se procedió al tatuaje intraoperatorio de la areola (7\%) (Fig. 8). Por último, en 5 casos no se pudo identificar el procedimiento.

La sucesión de complicaciones fue estudiada igualmente por la revisión de historias clínicas $(n=4)$. Tres pacientes sufrieron necrosis del pezón, en 2 de los casos parcial y solamente en uno de los casos total (5\%). Otra complicación posible, experimentada por una de las pacientes, fue la dehiscencia de la zona donante, que se trató con un injerto de piel total tomado de zona inguinal.

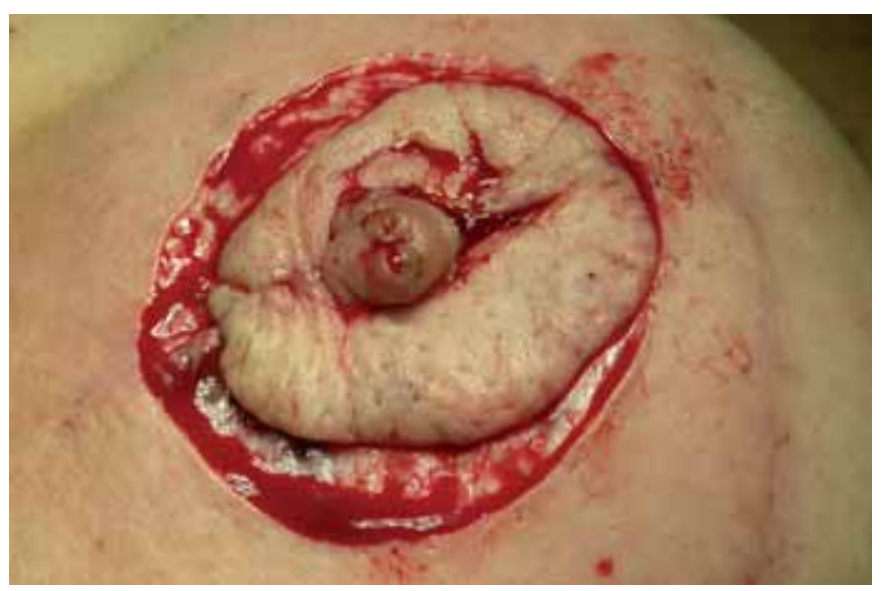

Fig. 6. Autoinjerto areolar. 


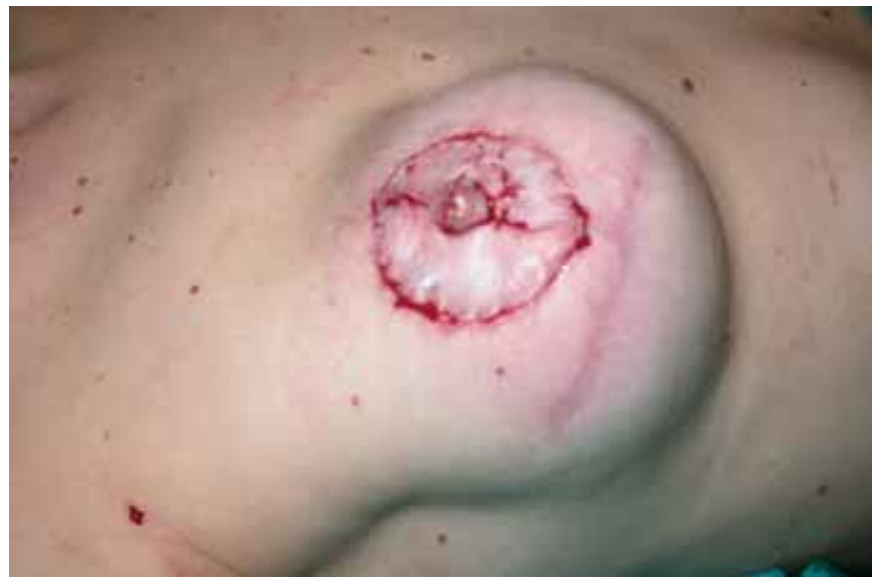

Fig. 7. Postoperatorio inmediato de reconstrucción con colgajo local de pezón y autoinjerto de areola.

Respondiendo a la pregunta de cuál era el aspecto que les gustaría cambiar acerca de su reconstrucción, en orden descendente, fue la falta/pérdida de proyección del pezón (Fig. 9), la forma y simetría del CAP, las cicatrices post-reconstrucción, la coloración de la areola, la textura, el tamaño y la posición. No obstante, es importante señalar que el $22 \%$ de las pacientes respondieron que no cambiarían nada acerca de su reconstrucción.

La satisfacción acerca de la reconstrucción del montículo mamario fue excelente o buena para el $68 \%$, normal para el $23 \%$ y pobre para el $9 \%$. En cambio, para la reconstrucción del complejo areola-pezón fue excelente o buena para el $50 \%$, normal para el $45 \%$ y pobre para el $5 \%$. (Fig. 10 y 11 ).

Teniendo en cuenta la valoración de las pacientes acerca de la reconstrucción del CAP no se encontraron diferencias significativas en los diferentes grupos en función del tiempo transcurrido entre el momento de la mastectomía y el tercer tiempo de la reconstrucción $(\mathrm{p}=0,06)$. Las pacientes que calificaron la reconstrucción del CAP como buena tuvieron un tiempo de espera medio de 31 meses, frente a los 10 meses de los que la calificaron como normal y a los que la calificaron como pobre cuya espera media fue de 24 meses. Agrupándose todas las pacientes, el tiempo de espera medio entre ambas intervenciones fue de 14 meses con una media de 8,5 meses.

La satisfacción de la reconstrucción del complejo areola pezón basándonos en el tipo de reconstrucción del pezón fue evaluada por test de Student. La técnica de la donación contralateral de pezón fue la que ofreció una mayor satisfacción (2,67 puntos) y proyección (7,23 puntos), seguida por las reconstrucciones mediante colgajos locales ( 2,26 y 5,85 puntos respectivamente). A pesar de las diferencias en sus medias, no se demostró ninguna diferencia estadísticamente significativa entre las diferente técnicas usa-

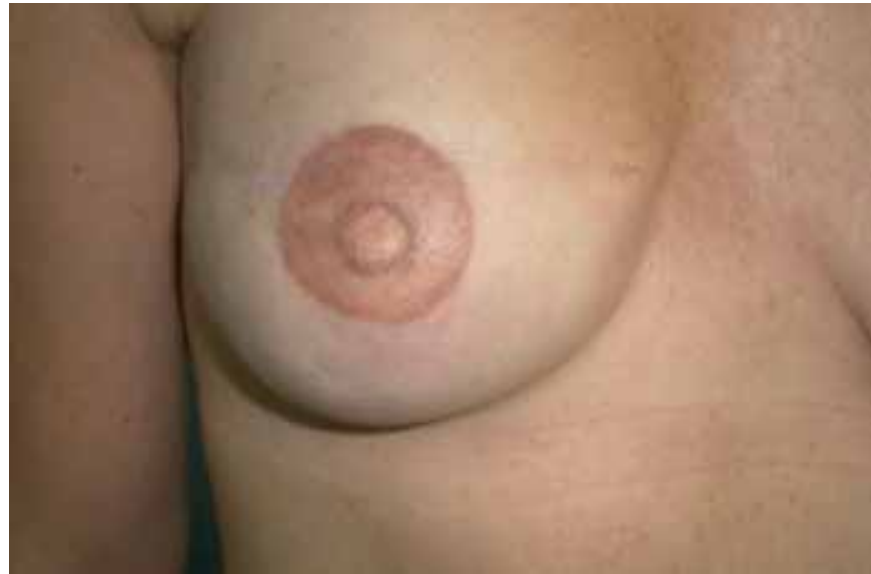

Fig. 8 Reconstrucción definitiva CAP mediante tatuaje, tras 1 año de evolución.

das valorándose el grado de satisfacción $(\mathrm{p}=0,09)$ y la puntuación de la proyección $(\mathrm{p}=0,44)$.

Teniendo en cuenta la técnica usada para la reconstrucción de la areola, la técnica de la donación-injerto de piel inguinal fue la que ofreció mayor satisfacción (3 puntos) y coloración (8,57 puntos), seguida de la técnica de la donación contralateral (2,29 y 6,30 puntosrespectivamente). La técnica del autoinjerto de areola aportó un grado de satisfacción de 2,26 puntos y una puntuación de la coloración de 6,13 puntos. A través del estudio estadístico se objetivaron diferencias estadísticas significativas de acuerdo al grado de satisfacción $(\mathrm{p}=0,01)$.

Por medio del estudio estadístico mediatne test de Student se comparó el grado de satisfacción final del montículo mamario contra la reconstrucción de la areolapezón. La satisfacción de la reconstrucción del montículo mamario tuvo una media de 2,47 puntos (Desviación Standard DS 0,65), mientras que la de la reconstrucción del CAP fue de 2,37 puntos (DS 0,57). Estas diferencias no fueron estadísticamente significativas $(\mathrm{p}=0,48)$.

A la hora de valorar los distintos aspectos de la reconstrucción mamaria, las medias de las puntuaciones fueron las siguientes: satisfacción con la reconstrucción final 8,32 puntos; satisfacción con el montículo mamario 8,32 puntos; proyección del CAP 6,95 puntos; desvanecimiento de su CAP 7,28 puntos; apariencia desnuda 7,5 puntos; apariencia con sujetador 8,14 puntos; simetría desnuda 6,81 puntos; simetría con sujetador 7,95 puntos. Por tanto, los aspectos más importantes a mejorar por orden de importancia serían la simetría desnuda, la proyección del pezón y el color de la areola.

Quizá uno de los datos más importantes de los obtenidos fue objetivar que a la pregunta de si la reconstrucción del CAP es absolutamente necesaria, el $75 \%$ de las encuestadas reconocieron que era algo fundamental para concluir una buena reconstrucción mamaria. 


\section{Discusión}

La reconstrucción del complejo areola-pezón ha sido infravalorada durante mucho tiempo, dándose tan solo importancia a la reconstrucción del montículo mamario. En los últimos tiempos distintos artículos han demostrado que el grado de satisfacción de las pacientes es mayor si se procede también a la reconstrucción del CAP $(3,4)$. Por ello, la reconstrucción tanto de la areola como del pezón es ofrecida a todas las pacientes mastectomizadas que van a llevar a cabo la reconstrucción de la mama inmediata o diferida en nuestro Servicio.

El objetivo del presente estudio fue la evaluación del grado de satisfacción tanto conjuntamente del grupo de pacientes a las cuales se les reconstruyó la areola y el pezón, como en función de la técnica reconstructiva utilizada para este cometido. Para ello se procedió a la revisión de historias clínicas y al diseño de un cuestionario. Con ese objetivo, se procedió a la revisión de distintos cuestionarios haciéndose un consenso entre los diferentes encontrados.

Toda esta información fue desarrollada para ver si había alguna diferencia en la escala de satisfacción en función de la técnica quirúrgica utilizada para la reconstrucción del CAP. Para ello, se procedió al estudio por separado de las distintas técnicas utilizadas tanto para la reconstrucción del pezón como para la de la areola.

Así, revisando las diferentes técnicas para la reconstrucción del pezón y su asociación con la satisfacción de la paciente, no se encontraron diferencias estadísticamente significativas. De esto se concluye que ninguna de las técnicas descritas se reconoce como superior para este cometido.

Por el contrario, revisando las diferentes técnicas para la reconstrucción de la areola, sí se encontraron diferencias estadísticamente significativas, que traslucen que el mayor grado de satisfacción se obtuvo en las pacientes cuya areola fue reconstruida mediante injerto de piel inguinal. No obstante, una de las complicaciones descritas fue la dehiscencia de sutura de la zona donante. A pesar de ser algo infrecuente, no debe infravalorarse dicha posibilidad que podría alargar el tiempo final de la reconstrucción mamaria, lo que podría traducirse como un aumento de la morbilidad.

Atendiendo al número de procedimientos necesitados para la reconstrucción del CAP, éste parece muy bajo, dado que tan solo el 3\% de las pacientes requirieron de una segunda intervención. Todos lo casos (2 en total) que requirieron una segunda intervención fue por complicaciones necróticas del pezón que necesitaron de la revisión del mismo.

Entre los distintos aspectos que mostraban mayor disconformidad entre las pacientes, el más repetido

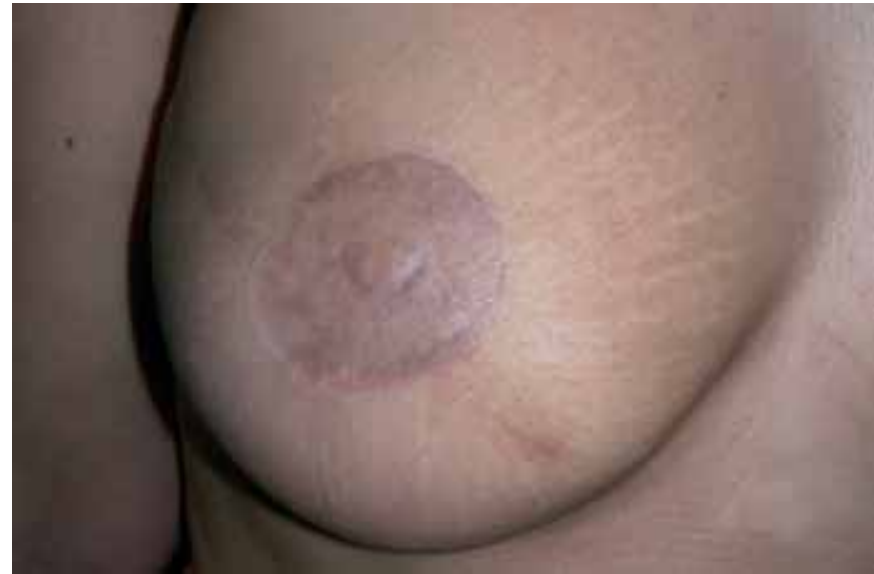

Fig. 9. Reabsorción- pérdida de proyección de pezón tras 1 año de evolución.

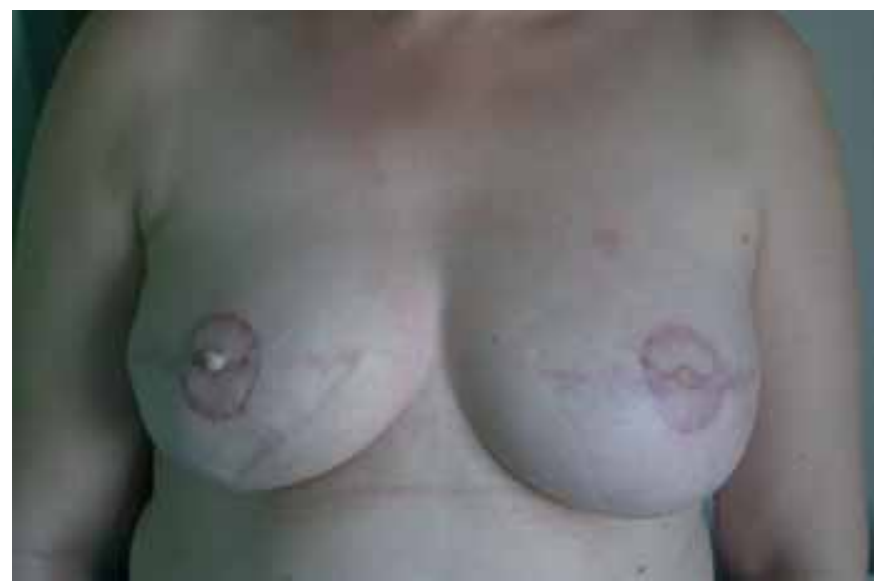

Fig. 10. Imagen postoperatoria definitiva mediante injerto piel inguinal tras 1 mes de evolución en reconstrucción bilateral.

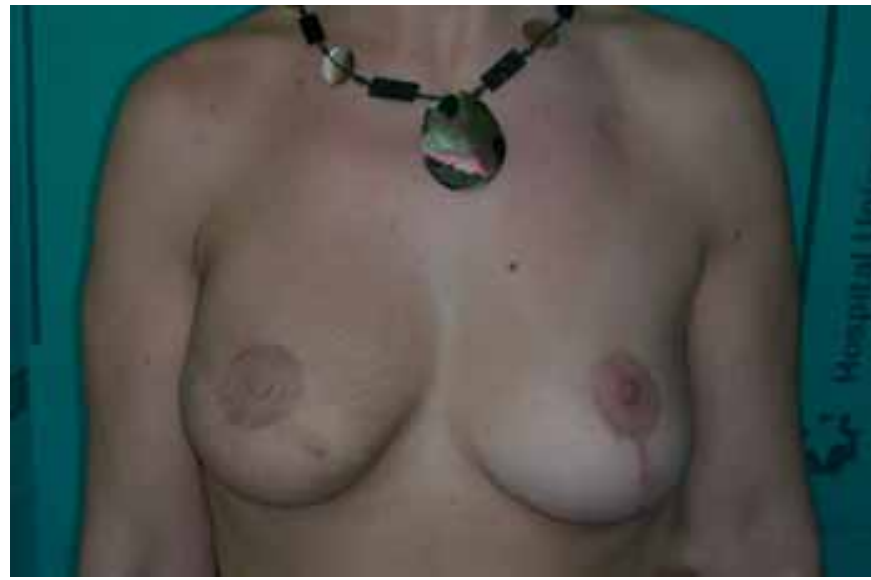

Fig. 11. Imagen postoperatoria definitiva mediante colgajo local, autoinjerto y tatuaje postoperatorio acompañado de pexia contralateral de 1 año de evolución.

fue la falta/pérdida de proyección del pezón, tal y como era de esperar por haberse descrito ya anteriormente por autores como Few (5) y Kroll (6). Sin embargo, dicha disconformidad fue seguida por un $25 \%$ de pacientes que no cambiarían nada acerca de su reconstrucción. A pesar de que el desvanecimiento del complejo ocupó un cuarto lugar, a todas las 
pacientes que se encontraban disconformes con la coloración obtenida y a aquellas a las cuales el cirujano responsable denotaba una posibilidad de mejoría, se les ofreció la posibilidad del tatuaje del mismo.

El tiempo transcurrido entre las distintas intervenciones quirúrgicas no fue algo que influyó en el grado de satisfacción. Dicho acontecimiento fue algo inesperado, pues contradice lo obtenido por otros autores (1). Así pues se puede concluir llegado a este resultado, que pacientes reconstruidas relativamente mucho tiempo atrás, se encuentran igualmente agradecidas una vez practicada la reconstrucción del CAP, a pesar de haber identificado en una de sus mamas la ausencia de éste. De ello se deduce que la reconstrucción del complejo areola pezón debe ofrecerse a todas las pacientes a pesar del tiempo de latencia desde la mastectomía.

A la hora de comparar el grado de satisfacción general, éste fue mayor con la reconstrucción del montículo mamario que del CAP. De este acontecimiento se puede concluir que uno de los aspectos que quedan por mejorar es la reconstrucción tanto de la areola como del pezón. Dado que las pacientes apuntaron que la reconstrucción del CAP es algo fundamental, el cirujano debe concienciarse de que la reconstrucción del CAP es uno de los aspectos pendientes para alcanzar la reconstrucción mamaria ideal.

\section{Conclusiones}

La reconstrucción del complejo areola pezón es un aspecto fundamental para completar una buena reconstrucción de mama y posiblemente sea la asignatura pendiente de los cirujanos plásticos. Entre las distintas técnicas de reconstrucción no hay ninguna cuyos resultados se sobrepongan a las demás, pero sí una vez que se reconstruye la areola, siendo la técnica que más satisface, el injerto inguinal. A pesar de ello, el aspecto más notable a mejorar lo encontramos a la hora de reconstruir el pezón, dada la frecuente disconformidad de las pacientes con la proyección conseguida a lo largo del tiempo, dada la posibilidad de reabsorción del mismo.

\section{Dirección del autor}

\author{
Dr. Ruben Fernández García \\ C/ Pedro Rico $19-10^{\circ} \mathrm{D}$ \\ 28029 Madrid. España \\ e-mail: rubenfergar@hotmail.com
}

Bibliografía

1. Jabor M.A., Shayani, P., Collins, D.R.., Karas, T., and. Cohen, B.E. "Nipple-Areola Reconstruction: Satisfaction and Clinical Determinants". Plast Reconst Surg. 2002. 110(2):457.

2. Roldán P. Lozano J.A., Oroz J. "Treatment of the contralateral breast in breast reconstruction. Nipple-areola reconstruction". Anales Universidad Navarra. 2005: 28, Suplemento 2.

3. Wellisch, D. K., Schain, W. S., Noone, R. B., Little, J.W. "The psychological contribution of nipple addition in breast reconstruction”. Plast. Reconstr. Surg. 1987, 80: 699.

4. Asplund, O. "Nipple and areola reconstruction: A study of 79 mastectomized women". Scand J Plast Reconstr Surg. 1983, 17(3):233

5. Few, J. W., Marcus, J. R., Vasas, L. A., Aitken, M. E., Redding, J. "Long-term predictable nipple projection following reconstruction". Plast. Reconstr. Surg. 1999, 104: 1321.

6. Kroll, S. S. "Nipple reconstruction with the douple-opposing tab flap". Plast. Reconstr. Surg. 1999, 104: 511.

7. Dean, N. R., Neild, T., Haynes, J., Cooter, R. D. "Fading of nipple-areolar reconstructions: the last hurdle in breast reconstruction?". Br. J. Plast. Surg. 2002, 55: 574.

8. Gruber, R. P. "Nipple-areola reconstruction: A review of techniques. "Clin. Plast. Surg" 1979, 6: 71.

9. Bhatty, M. A. and Berry, R. B. "Nipple-areola reconstruction by tattooing and nipple sharing". Br. J. Plast. Surg. 1997, 50: 331.

10. Draper, L.B., Bui, D.T., Chiu, F.S.: "Nipple-Areola reconstruction following chest-wall irradiation for breast cancer, Is it safe?". Ann Plast Surg 2005; 55: 12

11. Skillman, J. M., Ahmed, O., Dhearnsa B., Rowwell, A. R. "The Marshall technique: an economic one-stage technique for nippleareola reconstruction". Br J Plastic Surg 2002, 55: 504.

12. Dean, N., Haynes, J., Brennan, J., Neild, T., Goddard, C., Dearman, B., Cooter, R. "Nipple-areolar pigmentation: histology and potencial for reconstitution in breast reconstruction". Br J Plast Surg. 2005;58(2):202. 


\section{Comentario al trabajo uReconstrucción del complejo areola-pezón: revisión de 60 casosı}

\section{Dr. J osé Ángel Lozano Orella. Cirujano Plástico. Hospital Virgen del Camino. Pamplona (España)}

En primer lugar felicitar a los Drs. Fernández García, Fernández Delgado, Martínez, Bravo, García, Sordo y Casado por su trabajo y buenos resultados obtenidos. Coincido plenamente en la importancia de la recreación del complejo areola-pezón (CAP), que juega un papel integral dentro de un proceso tan complicado como es la reconstrucción de mama. Quiero recordar que este procedimiento no sólo supone conseguir un nuevo órgano con más o menos complejidad quirúrgica o mayor o menor morbilidad, sino alcanzar un equilibrio con la mama contralateral y, en definitiva, obtener una simetría "cuasi" perfecta (1).

El alto grado de satisfacción de la mujer con la reconstrucción del complejo areola-pezón está bien documentado en la literatura (2) y su indicación más que probada. Se han descrito un elevado número de técnicas quirúrgicas, lo que indica que ninguna es plenamente satisfactoria. En cuanto al procedimiento de reconstrucción del pezón sorprende que los autores hayan obtenido sus mejores resultados con el injerto de pezón contralateral, ya que, en principio, es una de las técnicas que sufre mayor grado de retracción. Todos sabemos que conseguir una proyección mantenida en el tiempo sin invaginaciones no es tarea fácil. Para evitarlo se han publicado sostenes de dermis (3), injertos de grasa, injertos de cartílago costal... pero es cierto que algo siempre retrae. En mi experiencia se obtienen buenos resultados mantenidos en el tiempo y retracciones mínimas con la técnica de la raya de Little (4). Se trata de una técnica sencilla que obtiene unos resultados perdurables en el tiempo y aplicable a cualquier tipo de reconstrucción mamaria.

Obtener una buena simetría de ambas areolas en cuanto a forma y color es un tema pendiente dentro de nuestra Cirugía Reconstructiva. Coincido con los autores en la gran utilidad del injerto cutáneo de la región inguinal. También estamos utilizando injertos de cicatriz residual de la mastectomía (5) e injertos de la areola contralateral, siempre que ésta sea grande. Estas técnicas evitan complicaciones de la zona donante inguinal. Pero lo que me parece definitivo en cuanto a la obtención de una buena simetría es el tatuaje bilateral de ambas areolas (6), tanto de la sana como de la reconstruida. Consigue lograr un color similar en ambos lados y permite corregir ciertas asimetrías de forma. En cuanto a su procedimiento, después de probar diferentes técnicas de pigmentación, hemos llegado a la conclusión de que

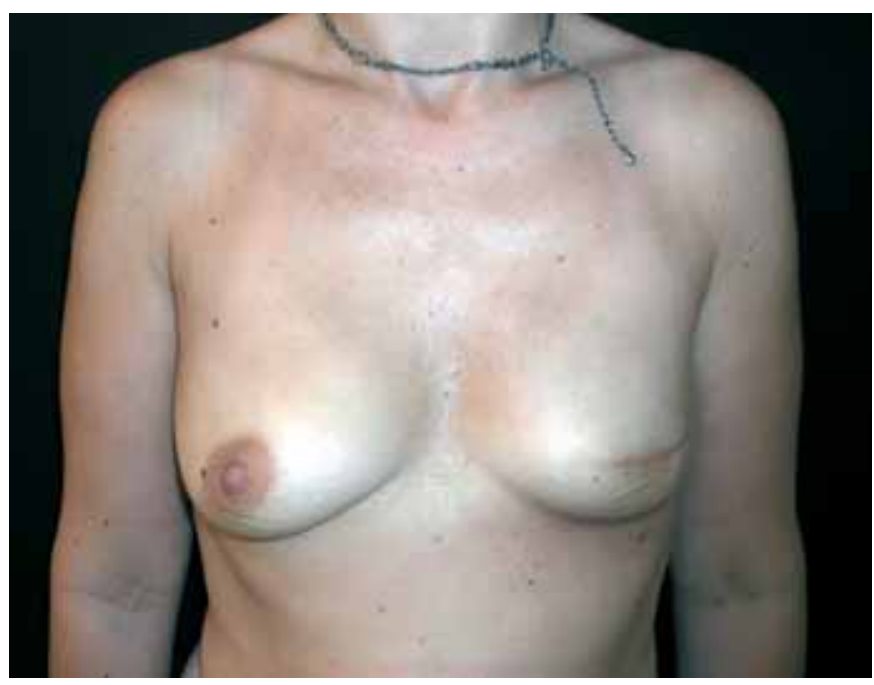

Fig. 1: Reconstrucción mamaria. el tatuaje debe ser realizado por profesionales (7) en un centro especializado para alcanzar un resultado plenamente satisfactorio.

Me gustaría hacer un último apunte sobre el tiempo de demora entre la mastectomía y la reconstrucción del CAP de 14 meses de media que presentan los autores. Es cierto que dentro de la Sanidad Pública es difícil buscar un hueco para este tipo de patología de pequeña envergadura, pero no se debiera demorar más de 6 meses (8), ya que si no las pacientes pueden caer en el olvido y, en definitiva, no reconstruirse completamente. Hay autores que defienden enérgicamente la reconstrucción inmediata del CAP para evitar este problema. Yo soy partidario de esperar un poco a que las mamas cojan su forma definitiva, sobre todo si se han empleado diferentes técnicas en cada una de ellas.

No me queda más que felicitar de nuevo a los autores coincidiendo en que la recreación del CAP es un paso tan importante como cualquier otro y necesario en la reconstrucción mamaria integral.

Bibliografía

1. Lozano JA, Escudero FJ, Colás C.: "Breast reconstruction with microsurgical perforator flaps". An Sist Sanit Navar. 2005;28 Suppl 2:73-79.

2. Shaikh-Naidu N, Preminger A, Rogers K, Messina P, Gayle L.: "Determinants of aesthetic satisfaction following TRAM and implant breast reconstruction". Ann Plast Surg 2004;52:465.

3. Lebeau J, Lopes TR, Gallodoro A, Raphael B.: "Nipple reconstruction: technical aspects and evolution in 14 patients". Plast Reconstr Surg. 2006 Mar;117:751.

4. Little JW III.: "Nipple-areola reconstruction". Clin Plast Surg 1984;11:351

5. Di Benedetto G, Sperti V, Pierangeli M, Bertani A.: "A simple and reliable method of nipple reconstruction using a spiral flap made of residual scar tissue". Plast Reconstr Surg. 2004;114:158.

6. El-Ali K, Dalal M, Kat CC.: "Tattooing of the nipple-areola complex: review of outcome in 40 patients". J Plast Reconstr Aesthet Surg. 2006;59:1052.

7. Fourie le R, Bruce-Chwatt.: "A Professional tattooing; alternative method to nipple reconstruction". Br J Plast Surg. 2004;57:693.

8. Farhadi J, Maksvytyte GK, Schaefer DJ, Pierer G, Scheufler O.: "Reconstruction of the nipple-areola complex: an update". J Plast Reconstr Aesthet Surg. 2006;59:40.

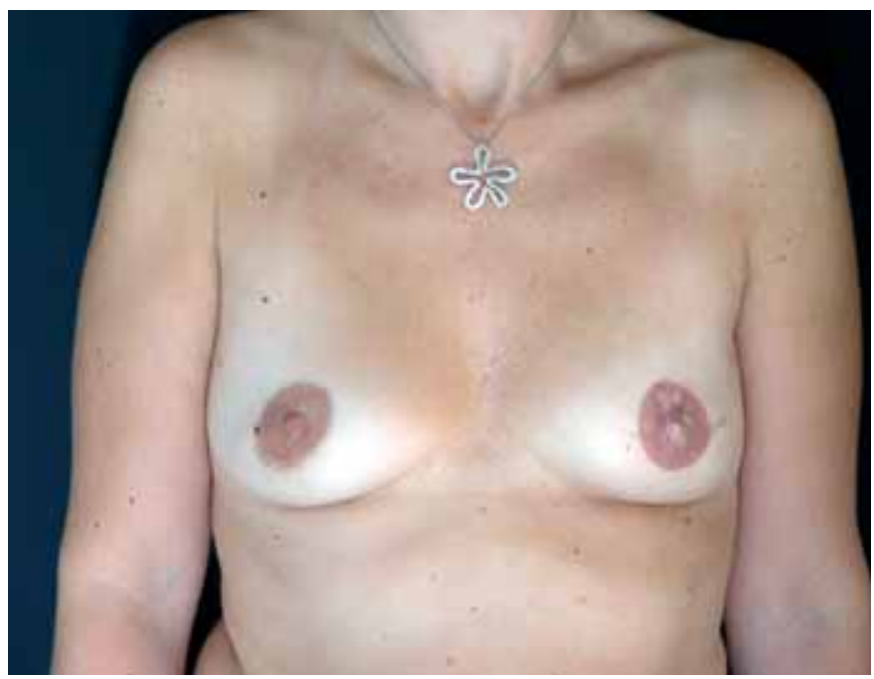

Fig. 2: Técnica de Little con tatuaje bilateral de ambas areolas. 


\section{Respuesta al comentario del Dr. José Ángel Lozano Orella}

\section{Dr. Rubén Fernández-García}

En primer lugar, agradecer los comentarios del Dr. Lozano Orella y tan solo aclarar determinados aspectos.

En efecto, sí que puede estar en lo cierto en que 14 meses de media hasta la reconstrucción definitiva desde el momento de la mastectomía pueden parecer demasiados, pero le indico que uno de los datos más reseñables de los obtenidos en el presente estudio fue que el factor tiempo no determina el grado de satisfacción de las pacientes.
Por último, destacar que el mayor número de reconstrucciones se hace mediante técnicas con colgajos locales, pero el estudio estadístico indicó que el injerto de pezón contralateral tiene unos resultados que lo avalan en sus restringidas indicaciones, como puede ser un pezón contralateral voluminoso, por lo que sí que es aplicable en determinados casos.

Sin más, agradezco sus observaciones y son bien recibidas sus felicitaciones. 